\title{
Renal outcomes in Asian patients with type 2 diabetes mellitus treated with SGLT2 inhibitors: a systematic review and meta-analysis of randomized controlled trials
}

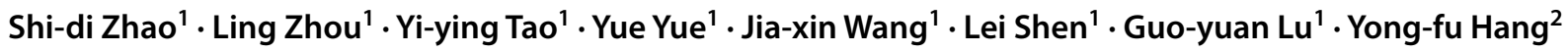

Received: 17 January 2021 / Accepted: 23 August 2021 / Published online: 14 September 2021

(C) The Author(s) 2021

\begin{abstract}
Aim This study investigated the effects of sodium-glucose cotransporter 2 (SGLT2) inhibitors on renal outcomes in Asian patients with type 2 diabetes mellitus (T2DM).

Materials and methods We searched Medline, EMBASE, and the Cochrane Library to identify randomized controlled trials published up to April 2020 that compared SGLT2 inhibitors with placebo or active comparator and reported any renal outcomes in Asian patients with T2DM. Random effects models and inverse variance weighting were used to calculate relative risks with 95\% confidence intervals (CIs).

Results We included 14 studies, totaling 3792 patients, in the analysis. In the short term, SGLT2 inhibitors significantly slowed estimated glomerular filtration rate (eGFR) decline (MD: 0.80; 95\% CI: 0.66 to $0.94 ; p<0.00001$ ) and reduced Scr levels (SMD: $-0.17 ; 95 \%$ CI: -0.23 to $-0.10 ; p<0.00001$ ) as compared with the control groups. The SGLT2 inhibitor group also had an advantage over the control group in lowering uric acid (UA) (SMD: $-1.2 ; 95 \% \mathrm{CI}:-1.30$ to $-1.11 ; p<0.00001$ ). There was no significant difference in urinary albumin creatinine ratio (UACR) reduction between the SGLT2 inhibitor and control groups (MD: $-8.87 ; 95 \% \mathrm{CI}:-19.80$ to $2.06 ; p=0.11$ ). However, dapagliflozin does appear to reduce albuminuria $(p=0.005)$. Lastly, SGLT2 inhibitors increased the incidence of adverse events (AEs) related to renal function (OR: 1.90; 95\% CI: 1.24 to $2.91 ; p=0.003$ ), but did not increase the incidence of renal impairment (OR: $0.85 ; 95 \%$ CI: 0.40 to $1.81 ; p=0.68$ ). Conclusion The use of SGLT2 inhibitors in Asian patients with T2DM can help delay the decline of eGFR and reduce Scr and UA. Although SGLT2 inhibitors have no overall advantage in reducing albuminuria, dapagliflozin does appear to reduce albuminuria, and while they may increase the occurrence of AEs related to renal function, they do not increase the incidence of renal impairment.
\end{abstract}

Keywords Type 2 diabetes mellitus $\cdot$ SGLT2 inhibitors $\cdot$ Asian $\cdot$ Renal outcome $\cdot$ Meta-analysis

\section{Introduction}

According to the International Diabetes Federation (IDF), the number of patients with diabetes reached 451 million worldwide in 2017. Among them, Asians account for the largest proportion [1]. Diabetic kidney

Ling Zhou

z166060@163.com

1 Department of Nephrology, The First Affiliated Hospital of Soochow University, Suzhou 215006, People's Republic of China

2 Department of Pharmacy, The First Affiliated Hospital of Soochow University, Suzhou 215006, People's Republic of China disease (DKD) is one of the most common and serious complications of diabetes [2]. Studies have shown that Asian people with type 2 diabetes (T2DM) have unique clinical presentations and are more likely to have DKD [3].

Sodium-glucose cotransporter 2 (SGLT2) inhibitors reduce the glucose reabsorption capacity of the kidney by inhibiting glucose transporters on the surface of the proximal tubules of the kidney, thereby promoting the excretion of urinary glucose [4]. In recent years, studies from across the globe have shown that SGLT2 inhibitors are beneficial to the cardiovascular and renal outcomes of patients with T2DM [5]. However, although Asians are at high risk of DKD, meta-analyses focused on this population are lacking. Therefore, we performed a systematic review and 
meta-analysis of randomized controlled trials (RCTs) to investigate the effects of SGLT2 inhibitors on renal outcomes in Asian patients with T2DM.

\section{Methods}

\section{Study design}

This study is a systematic review and meta-analysis that evaluated the effects of SGLT2 inhibitors on the renal outcomes of Asian patients with T2DM as compared with placebo or positive controls. We collected randomized controlled studies related to SGLT2 inhibitors, included studies compared with placebo and active comparator (antidiabetic drugs). Other prospective and nonrandomized studies were excluded.

\section{Data sources and searches}

We searched Medline, EMBASE, and the Cochrane Central Register of Controlled Trials for RCTs of SGLT2 inhibitors published as full articles and until April 2020. The search terms used for SGLT2 inhibitors were "sodium-dependent glucose transporters 2," "SGLT2 inhibitor," or "SGLT-2 inhibitor." The retrieval process is shown in Fig. 1.

\section{Study selection}

We included all studies that reported individual RCT data of any SGLT2 inhibitors versus placebo or active controls in Asian patients with T2DM. We eliminated duplicate

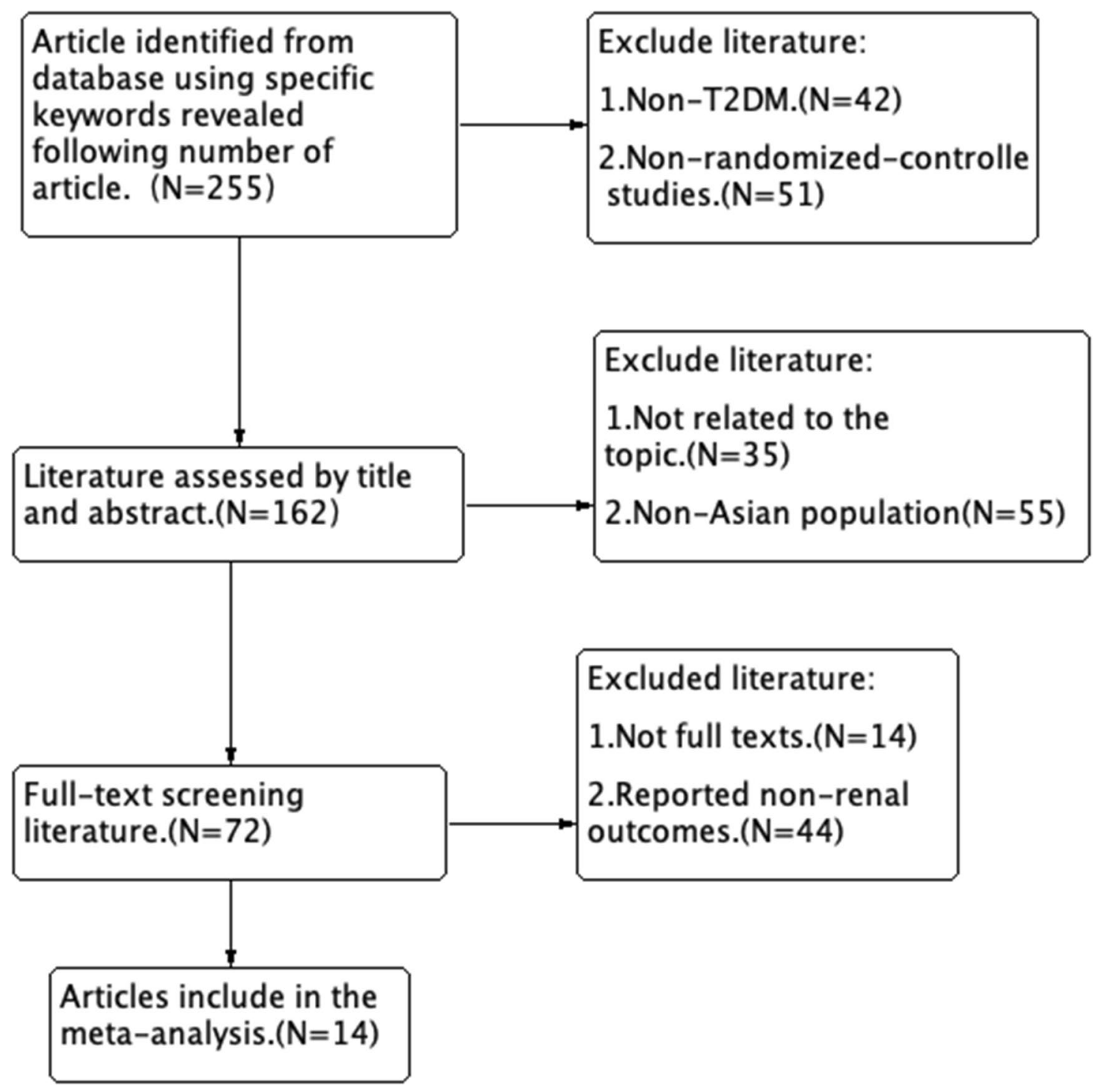

Fig. 1 Identification of eligible studies 
Table 1 Baseline characteristics of the studies included in the meta-analysis

\begin{tabular}{|c|c|c|c|c|c|c|c|c|}
\hline \multirow[t]{2}{*}{ Study } & \multirow{2}{*}{$\begin{array}{l}\text { Interventions } \\
\text { (dose of } \\
\text { SGLT2i) }\end{array}$} & \multicolumn{2}{|l|}{ Control } & \multicolumn{2}{|l|}{$\begin{array}{l}\text { Number of } \\
\text { patients }\end{array}$} & \multirow{2}{*}{$\begin{array}{l}\text { Duration of } \\
\text { intervention, } \\
\text { weeks }\end{array}$} & \multirow{2}{*}{$\begin{array}{l}\text { Quality } \\
\text { assess- } \\
\text { ment }\end{array}$} & \multirow[t]{2}{*}{ Outcome } \\
\hline & & & & SGLT2i & Comp & & & \\
\hline Lu et al. [16] & $\begin{array}{l}\text { Ipragliflozin } \\
50 \mathrm{mg}\end{array}$ & Placebo & Metformin & 87 & 83 & 24 weeks & 5 & eGFR, Scr, UACR \\
\hline Kaku2014 & $\begin{array}{l}\text { Tofogliflozin } 10 \\
\mathrm{mg}, 20 \mathrm{mg} \\
40 \mathrm{mg}\end{array}$ & Placebo & & 165 & 55 & 24 weeks & 5 & eGFR, Scr, UA \\
\hline Kaku2014 2 & $\begin{array}{l}\text { Dapagliflozin } \\
5 \mathrm{mg}, 10 \mathrm{mg}\end{array}$ & Placebo & & 174 & 87 & 24 weeks & 4 & Renal impairment \\
\hline Han et al. [13] & $\begin{array}{l}\text { Ipragliflozin } \\
50 \mathrm{mg}\end{array}$ & Placebo & Metformin & & & & & \\
\hline Sitagliptin & 73 & 66 & 24 weeks & 5 & eGFR, Scr & & & \\
\hline Ji et al. [15] & $\begin{array}{l}\text { Dapagliflozin } \\
5 \mathrm{mg}, 10 \mathrm{mg}\end{array}$ & Placebo & & 261 & 132 & 24 weeks & 5 & $\begin{array}{l}\text { eGFR, Scr, UA, } \\
\text { renal impair- } \\
\text { ment }\end{array}$ \\
\hline Ji et al. [14] & $\begin{array}{l}\text { Canagliflozin } \\
100 \mathrm{mg}, \\
300 \mathrm{mg}\end{array}$ & Placebo & Metformin & & & & & \\
\hline $\begin{array}{l}\text { Metformin + sul- } \\
\text { phonylurea }\end{array}$ & 450 & 226 & 18 weeks & 5 & eGFR, Scr & & & \\
\hline Inagaki et al. [9] & $\begin{array}{l}\text { Canagliflozin } \\
100 \mathrm{mg}, \\
200 \mathrm{mg}\end{array}$ & Placebo & & 178 & 93 & 24 weeks & 5 & Scr, UACR \\
\hline Seino2014 & $\begin{array}{l}\text { Luseogliflozin } \\
2.5 \mathrm{mg}\end{array}$ & Placebo & & 79 & 79 & 24 weeks & 6 & $\begin{array}{l}\text { Scr, UA, AE } \\
\text { related to renal } \\
\text { function }\end{array}$ \\
\hline Seino $2014^{2}$ & $\begin{array}{l}\text { Luseogliflozin } \\
0.5 \mathrm{mg} 2.5 \mathrm{mg} \\
5 \mathrm{mg}\end{array}$ & Placebo & Diet therapy & 183 & 56 & 12 weeks & 4 & $\begin{array}{l}\text { Scr, UA, AE } \\
\text { related to renal } \\
\text { function }\end{array}$ \\
\hline Seino $2014^{3}$ & $\begin{array}{l}\text { Luseogliflozin } \\
1 \mathrm{mg}, 2.5 \mathrm{mg} \\
5 \mathrm{mg}, 10 \mathrm{mg}\end{array}$ & Placebo & & 224 & 58 & 12 weeks & 5 & $\begin{array}{l}\text { Scr, UA, AE } \\
\text { related to renal } \\
\text { function }\end{array}$ \\
\hline Yang et al. [7] & $\begin{array}{l}\text { Dapagliflozin } \\
10 \mathrm{mg}\end{array}$ & Placebo & $\begin{array}{l}\text { Insulin or oral } \\
\text { antihypergly- } \\
\text { cemic }\end{array}$ & 139 & 133 & 24 weeks & 4 & Renal impairment \\
\hline Yang et al. [19] & $\begin{array}{l}\text { Dapagliflozin } \\
5 \mathrm{mg}, 10 \mathrm{mg}\end{array}$ & Placebo & Metformin & 299 & 145 & 24 weeks & 6 & $\begin{array}{l}\text { AE related to } \\
\text { renal function }\end{array}$ \\
\hline $\begin{array}{l}\text { Terauchi et al. } \\
\text { [17] }\end{array}$ & $\begin{array}{l}\text { Tofogliflozin } \\
20 \mathrm{mg}\end{array}$ & Placebo & & 140 & 70 & 16 weeks & 6 & UA \\
\hline Bancha2019 & $\begin{array}{l}\text { Dapagliflozin } \\
10 \mathrm{mg}\end{array}$ & Control & & 28 & 29 & 12 weeks & 4 & $\begin{array}{l}\text { eGFR, Scr, } \\
\text { UACR, UA }\end{array}$ \\
\hline
\end{tabular}

The superscript numbers are used to distinguish between different randomized controlled studies with the same author

$e G F R$, estimated glomerular filtration rate; Scr, serum creatinine; UACR, urinary albumin/creatinine ratio; UA, uric acid; adverse events (AEs) related to renal function

publications of original RCTs and screened the titles and abstracts of identified studies to identify RCTs that reported at least one of the following renal outcomes: estimated glomerular filtration rate (eGFR), serum creatinine (Scr), urinary albumin creatinine ratio (UACR), uric acid (UA), adverse events (AEs) related to renal function, and renal impairment.

\section{Data extraction}

Two reviewers independently extracted the data using a predefined data extraction form. Disagreements were resolved by discussion with a third reviewer. Extracted data included the first author, study characteristics, experimental and control group treatments, and renal outcome(s). 
Fig. 2 Risk of bias graph

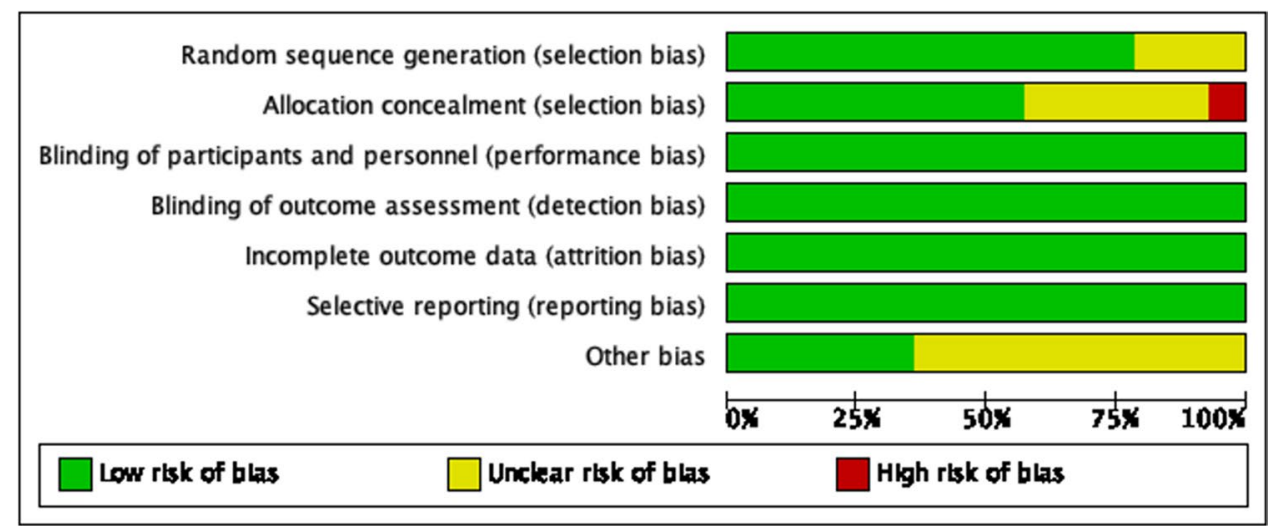

\section{Quality assessment}

The revised Jadad scale [6] was used to evaluate the methodological quality of the included literature.

\section{Data synthesis and analysis}

Statistical analyses were conducted with Review Manager (RevMan, version 5.4) software. Measures of treatment effect included mean differences (MDs), standardized mean differences (SMDs), odds ratios (ORs), and 95\% confidence intervals (CIs).

\section{Results}

\section{Characteristics of the included studies}

Fourteen articles were included in the study. All investigated the effects of different SGLT2 doses. We conducted subgroup analyses of the different SGLT2 drugs and treated each drug dose as an independent study. The drugs (doses) included ipragliflozin $(50 \mathrm{mg})$, tofogliflozin $(10 \mathrm{mg}, 20 \mathrm{mg}$, and $40 \mathrm{mg}$ ), dapagliflozin (5 $\mathrm{mg}$ and $10 \mathrm{mg}$ ), canagliflozin $(100 \mathrm{mg}, 200 \mathrm{mg}$, and $300 \mathrm{mg})$, and luseogliflozin $(0.5 \mathrm{mg}$, $1 \mathrm{mg}, 2.5 \mathrm{mg}, 5 \mathrm{mg}$, and $10 \mathrm{mg}$ ). The maximum follow-up time of the RCTs was 24 weeks. Table 1 presents further detail on the characteristics of the included studies.

In the meta-regression, five studies had a baseline eGFR of $60-89 \mathrm{~mL} / \mathrm{min} / 1.73 \mathrm{~m}^{2}$ [7-11], three studies had a baseline eGFR of $>89 \mathrm{~mL} / \mathrm{min} / 1.73 \mathrm{~m}^{2}$ [12-14], and the baseline eGFR of six studies was unknown [15-20].

\section{Assessment of study quality and risk of bias}

Each included study reported patients' baseline data and all mentioned "randomization" in the article. The range of the overall Jadad scores was $4-7$, with a relatively high correlation between quality and quantity. The risk bias graph is shown in Fig. 2, and the risk bias summary is shown in Fig. 3.

\section{Change in eGFR}

The level of eGFR was reported in 6 RCTs ( 5 placebo comparison and 1 active comparison trials) [10, 11 , 13-16]. Their baseline eGFR interval was primarily between 60 and $89 \mathrm{~mL} / \mathrm{min} / 1.73 \mathrm{~m}^{2}$, and all received double-blind treatment within 24 weeks. Analysis of these studies found that SGLT2 inhibitors significantly slowed the decline in eGFR as compared with controls (MD: 0.80 ; 95\% CI: 0.66 to $0.94 ; p<0.00001$; Fig. 4). However, this estimate is heavily weighted in studies using dapagliflozin. Dapagliflozin can delay the decline of eGFR, and the difference was statistically significant (MD: $0.81 ; 95 \% \mathrm{CI}: 0.67$ to 0.95 ; $p<0.00001$ ), while ipragliflozin, tofogliflozin, and canagliflozin had no significant difference compared with the control group. The funnel plot of comparison in eGFR is shown in Fig. 5.

\section{Changes in Scr and UA}

The level of Scr was reported in 10 RCTs (9 placebo comparison and 1 active comparison trials) $[9-16,18,20]$. Compared with the control group, SGLT2 inhibitor could reduce the level of Scr (SMD: -0.17 ; 95\% CI: -0.23 to -0.10 ; $p<0.00001$; Fig. 6). Dapagliflozin has the largest weight (SMD: $-0.96 ; 95 \%$ CI: -1.10 to $-0.82 ; p<0.00001$ ). However, tofogliflozin slightly increased Scr (SMD: 0.32; 95\% CI: 0.10 to $0.55 ; p=0.005$ ); ipragliflozin, canagliflozin, and luseogliflozin had no significant difference compared with the control group. The funnel plot of comparison in Scr is shown in Fig. 7. 


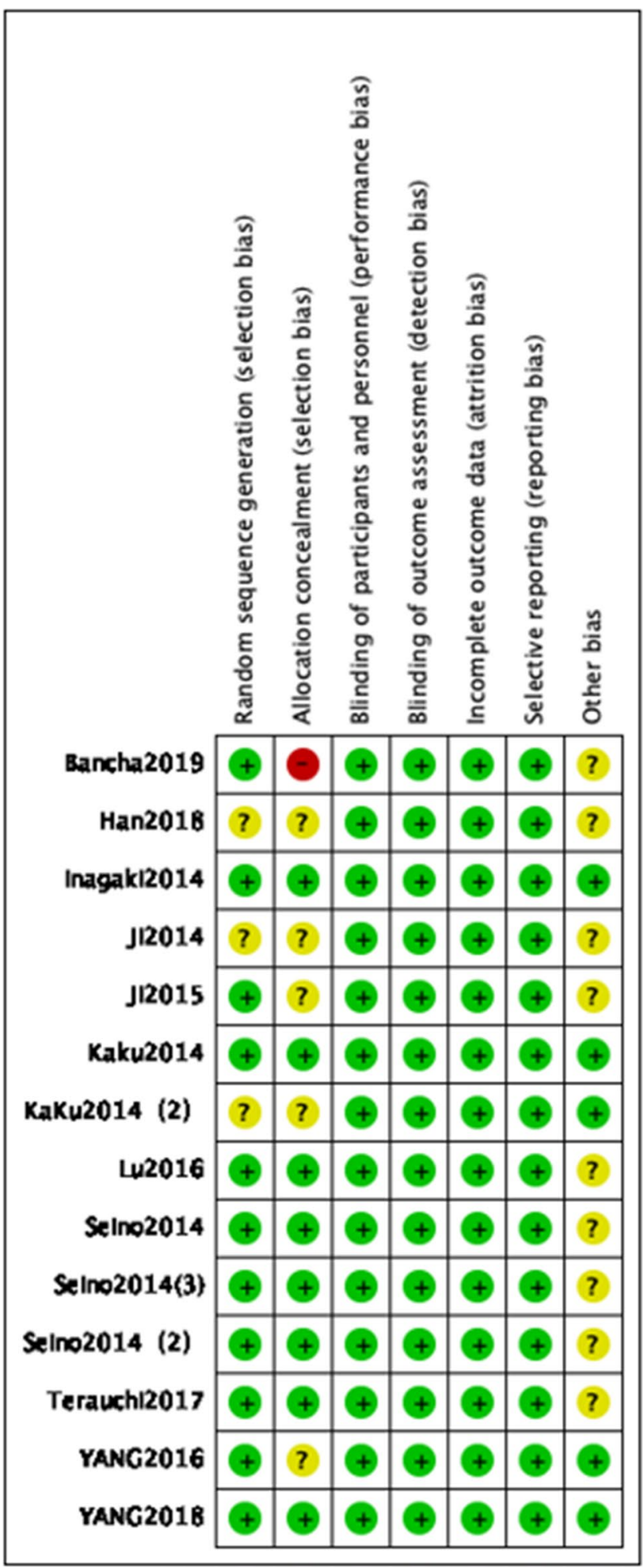

Fig. 3 Risk of bias summary

The level of UA was reported in 8 RCTs ( 7 placebo comparison and 1 active comparison trials) [7, 10-12, 15, $17,18,20]$. The paper found that SGLT2 inhibitors significantly reduced the level of UA (SMD: $-1.20 ; 95 \%$ CI: -1.30 to $-1.11 ; p<0.00001$; Fig. 8 ). Among them, luseogliflozin (SMD: $-0.55 ; 95 \%$ CI: -0.68 to $-0.42 ; p<0.00001$ ), dapagliflozin (SMD: -3.52 ; 95\% CI: -3.71 to -3.33 ; $p<0.00001$ ), and tofogliflozin (SMD: $-0.35 ; 95 \%$ CI: -0.52 to $-0.17 ; p=0.0001$ ) to reduce the levels of UA were statistically significant. The funnel plot of comparison in UA is shown in Fig. 9.

\section{Changes in UACR}

We used UACR to assess albuminuria. The level of UACR was reported in 3 RCTs ( 2 placebo comparison and 1 active comparison trials) $[9,11,16]$. There was no significant difference in the reduction of UACR between the SGLT2 inhibitor and control groups (MD: $-8.87 ; 95 \% \mathrm{CI}:-19.80$ to $2.06 ; p=0.11$; Fig. 10). However, dapagliflozin can reduce the level of UACR; the difference was statistically significant (MD: $-43.20 ; 95 \% \mathrm{CI}:-73.68$ to $-12.72 ; p=0.005$ ). Compared with the control group, canagliflozin and ipragliflozin had no significant difference in reducing UACR. The funnel plot of comparison in UACR is shown in Fig. 11.

\section{Effect on AEs related to renal function and renal impairment}

AEs related to renal function was reported in 4 RCTs (4 placebo comparison trials) $[12,18-20]$. Based on a predefined list of MedDRA preferred terms, AEs related to renal function include presence of albumin in urine, urinary calculus, presence of cells in urine, increased NAG, increased urinary b2 microglobulin, presence of blood in urine, presence of red blood cells in urine, and presence of white blood cells in urine, pollakiuria etc. [18, 19]. All AEs related to renal function were classified as mild and most of these resolved during the study. In the meta-analysis, SGLT2 inhibitors were found to increase the occurrence of AEs related to renal function as compared with the control group (OR: 1.90; 95\% CI: 1.24 to $2.91 ; p=0.003$; Fig. 12 ). Luseogliflozin increased the incidence of AEs related to renal function accounted for the largest weight (OR: 1.90; 95\% CI: 1.22 to $2.95 ; p=0.004$ ), while dapagliflozin had no significant difference in increasing AEs related to renal function compared with the control group (OR: 1.95; 95\% CI: 0.35 to $10.74 ; p=0.44)$. The majority of AEs had a low incidence and were mild in severity. The funnel plot of comparison in AEs related to renal function is shown in Fig. 13.

The renal impairment was reported in 3 RCTs (3 placebo comparison trials) $[7,8,15]$. Events of renal impairment were observed during within 24-week double-blind treatment period, and all the renal impairment events were 


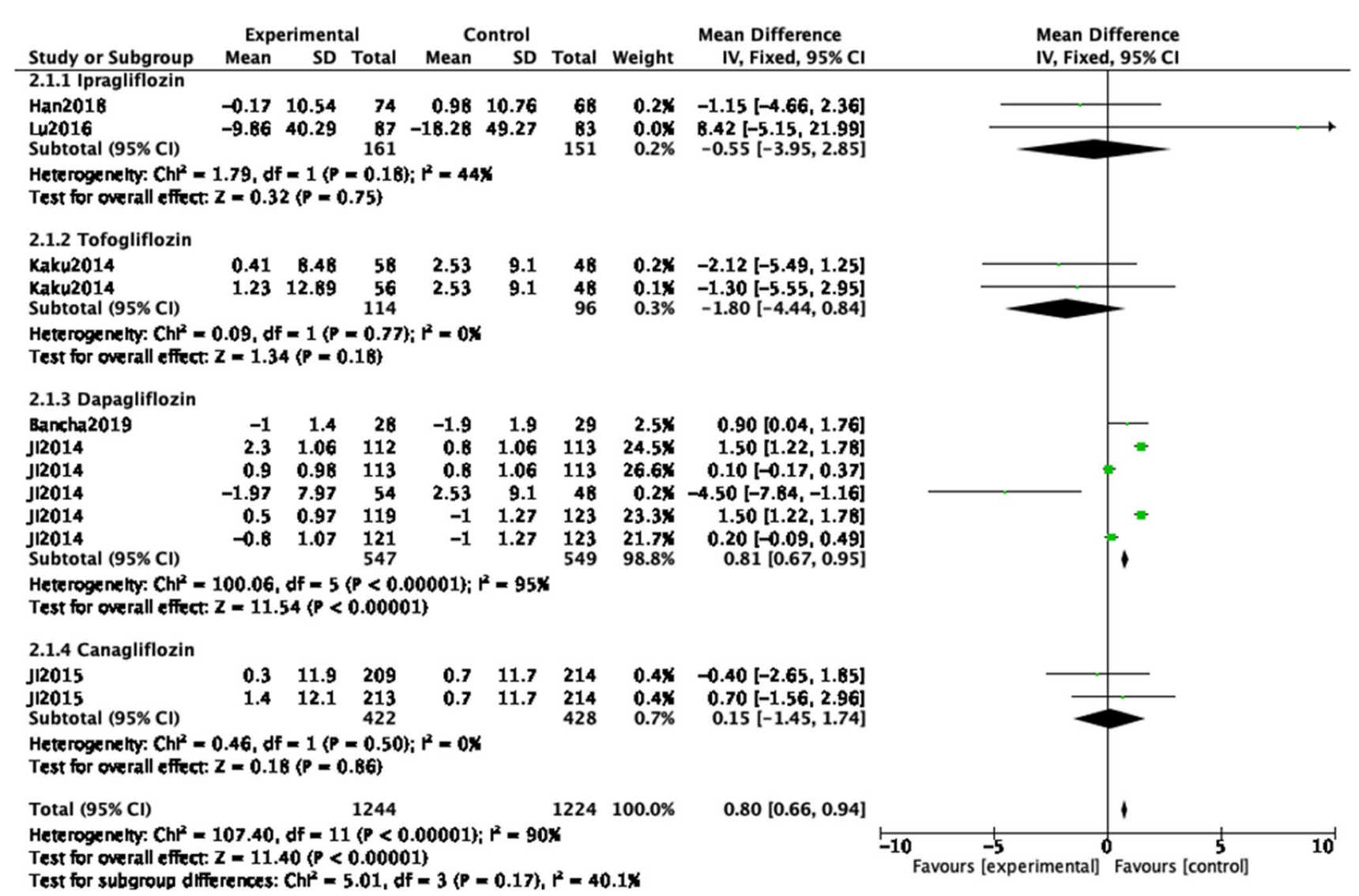

Fig. 4 Forest plot of the \% change in the eGFR in the patients randomized to SGLT2 inhibitor therapy versus control therapy. SD standard deviation, CI confidence interval, IV inverse variance

of mild or moderate intensity, and none were serious or resulted discontinuation. Compared with the control group, the SGLT2 inhibitor group did not increase the risk of renal impairment (OR: 0.85 ; $95 \%$ CI: 0.40 to $1.81 ; p=0.68$; Fig. 14). The funnel plot of comparison in the renal impairment is shown in Fig. 15.

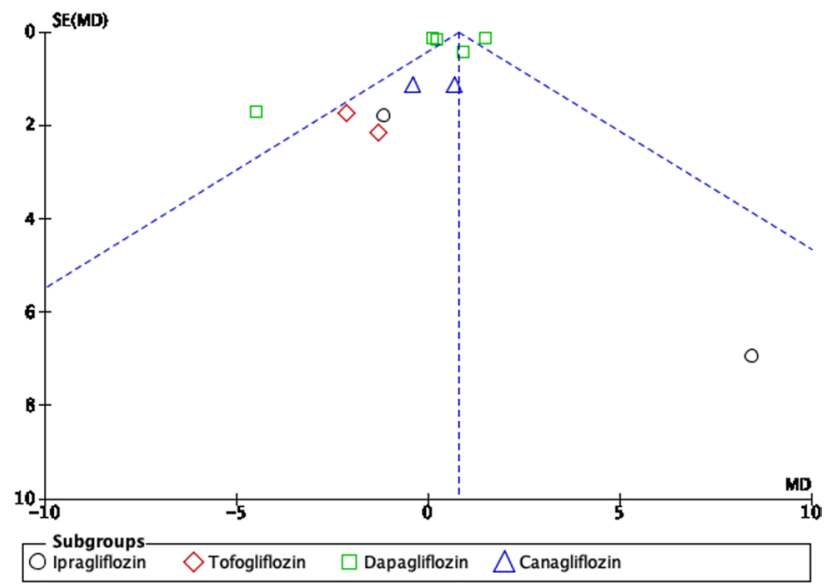

Fig. 5 Funnel plot of comparison in eGFR

\section{Discussion}

The diabetes epidemic and its complications pose a major global threat [1]; more than $90 \%$ of diabetic patients have T2DM [21]. With the increased incidence of T2DM, the incidence of DKD is also increasing annually [22]. As compared with non-Asian people, Asians have their own characteristics. They have a lower average body mass index, higher body fat content, and more visceral fat [23]. This difference between Asian and non-Asian populations is related to genetic susceptibility and eating habits. Someone found that the Asians diet contains more carbohydrates (e.g., white rice) [24]. Foods with a high glycemic index stimulate the pancreas to secrete a high demand for insulin. When the pancreas has congenital dysfunction, this leads to increased serum glucose levels [25]. A large multinational clinical study found that Asian diabetics are more likely to develop DKD [26]. The incidence of DKD in Asia, America, and the Caribbean is higher than other regions or countries [27]. The differences in the prevalence of DKD are due to many factors, including age, obesity, the course of diabetes, serum 


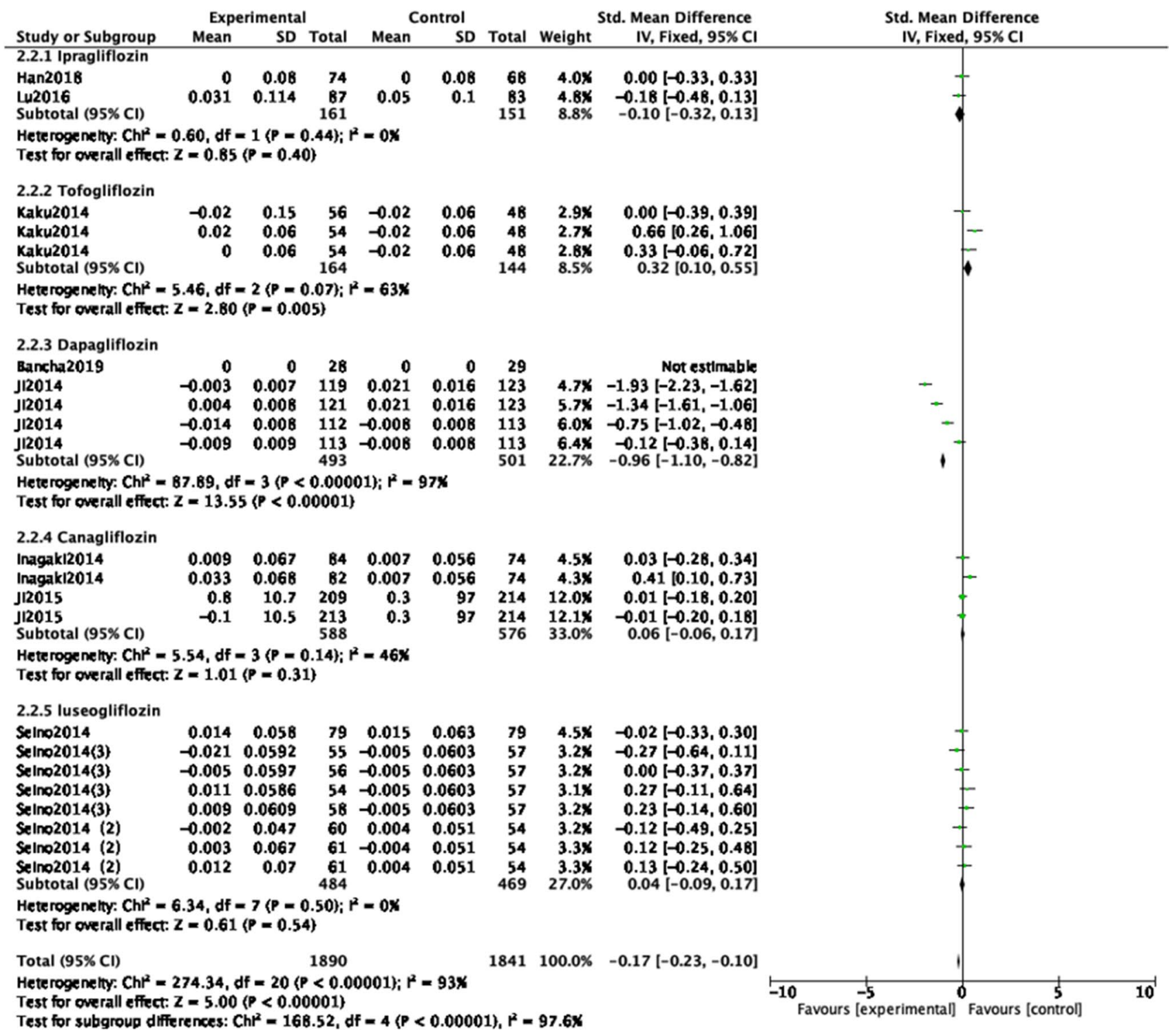

Fig. 6 Forest plot of the \% change in Scr in the patients randomized to SGLT2 inhibitor therapy versus control therapy. SD standard deviation, CI confidence interval, IV inverse variance

glucose, blood pressure, blood lipid levels, criteria used to diagnose DKD, and genetic factors [28].

SGLT2 inhibitors reduce the reabsorption of glucose by the kidney and make excessive glucose excreted from the urine, thereby lower plasma glucose levels [29]. This paper focused on the renal effects of SGLT2 inhibitors in Asian population. Existing studies have found that SGLT2 inhibitors can lower blood lipid level, reduce body weight, decrease systolic blood pressure, and reduce blood UA to slow the progression of DKD [30-33]. In addition, SGLT2 inhibitors have been shown to have beneficial effects on the kidneys. The DAPA-CKD clinical trial enrolled patients with eGFRs between 25 and $75 \mathrm{~mL} /$ $\mathrm{min} / 1.73 \mathrm{~m}^{2}$ and UACRs between 200 and $5000 \mathrm{mg} / \mathrm{g}$. It concluded that dapagliflozin may significantly reduce the risk of renal failure in patients with chronic kidney disease (CKD) [34].

In recent years, four large-scale clinical trials used decline in eGFR, end-stage renal disease, doubling of $\mathrm{Scr}$, or renal death as indicators of renal outcome [35-38]. The EMPA-REG OUTCOME study reported a significant relative risk reduction of $44 \%$ in the doubling of Scr and a $55 \%$ lower relative risk of the initiation of continuous renal-replacement therapy in the 


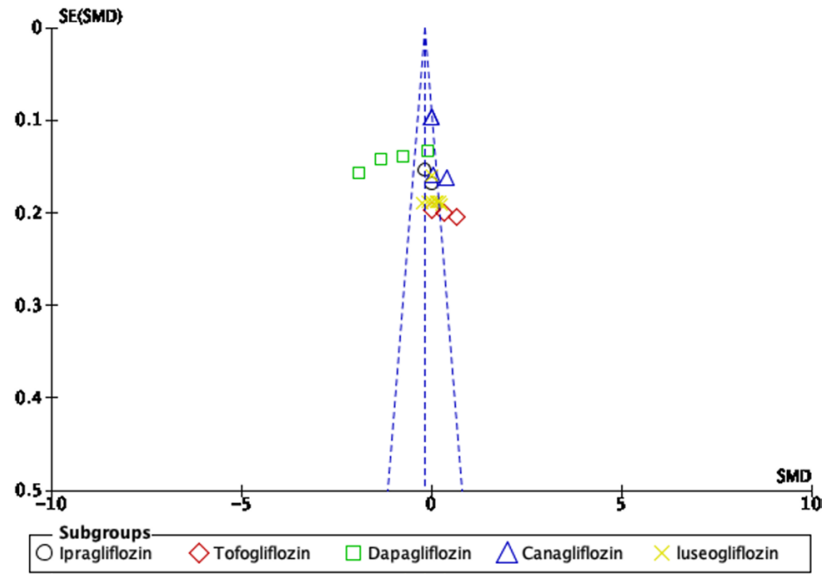

Fig. 7 Funnel plot of comparison in Scr

empagliflozin group as compared with the placebo group [36]. In the CANVAS study, canagliflozin significantly reduced the risk of the renal endpoint outcome [37]. In the DECLARE-TIMI 58 trial, canagliflozin was additionally found to reduce the incidence of adverse renal outcomes in patients with T2DM [35]. Finally, CREDENCE enrolled 4401 participants with a baseline eGFR of $30-90 \mathrm{~mL} / \mathrm{min} / 1.73 \mathrm{~m}^{2}$ and albuminuria (as indicated by a UACR of $300-5000 \mathrm{mg} / \mathrm{g}$ ). The trial found that during a median follow-up of 2.62 years, the risk of renal failure events in the canagliflozin group was lower than in the placebo group [38].

Although large clinical trials such as EMPA-REG, CANVAS, and DECLARE-TIMI 58 have confirmed the cardiovascular benefits of SGLT2 inhibitors [35-37], due to population differences, the benefits of SGLT2 inhibitors in Asian population need to be further studied. A metaanalysis focusing on Asians found that the use of SGLT2 inhibitors did not reduce the incidence of adverse cardiovascular events, hospitalization heart failure, or cardiovascular death [39]. As a result, we still lack meta-analysis of renal outcomes associated with SGLT2 inhibitors in Asian populations.

Our meta-analysis found that the short-term use of SGLT2 inhibitors in Asians may delay the decline of eGFR and reduce Scr and UA. These findings are

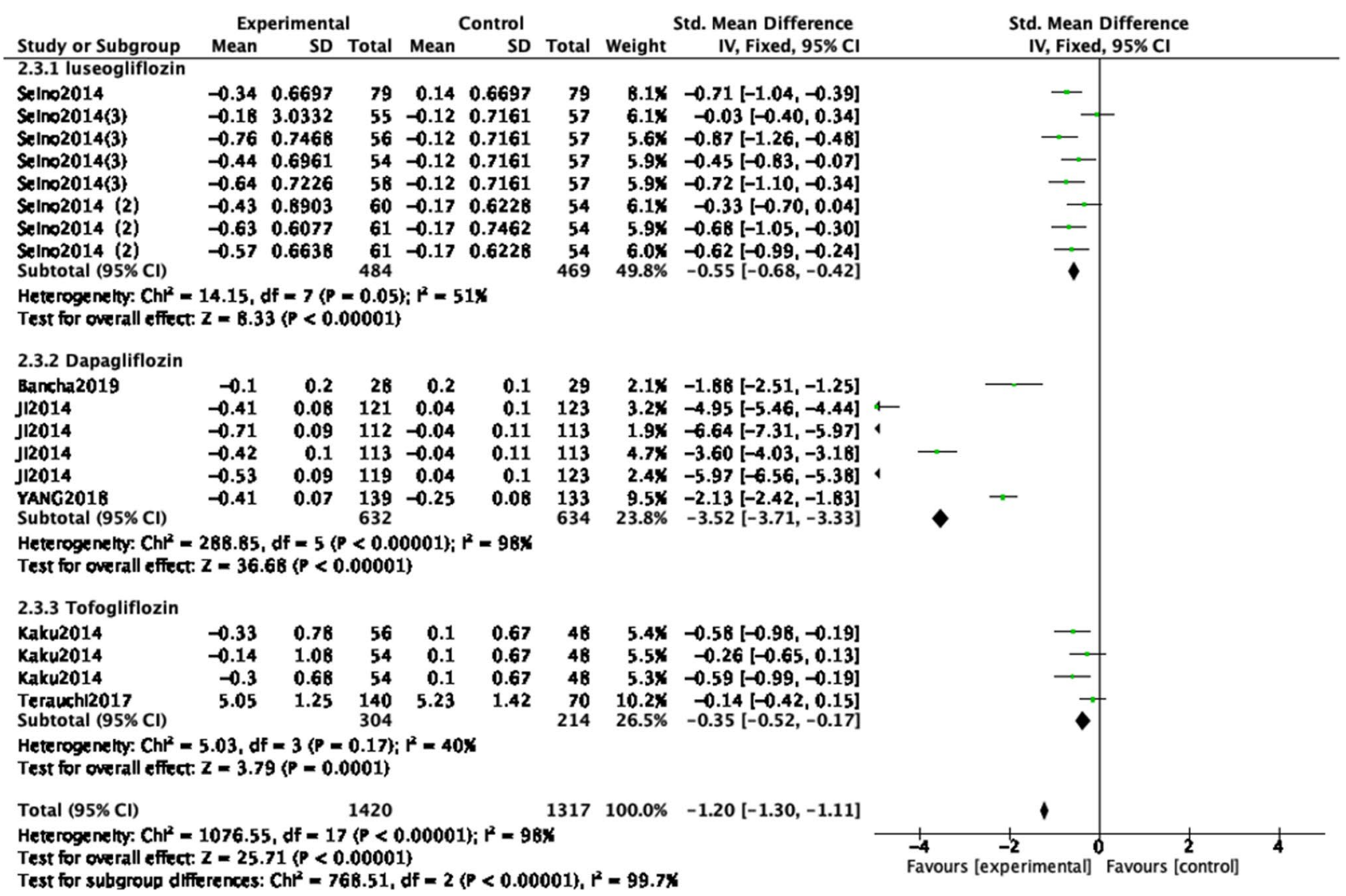

Fig. 8 Forest plot of the \% change in uric acid (UA) in the patients randomized to SGLT2 inhibitor therapy versus control therapy. SD standard deviation, CI confidence interval, IV inverse variance 


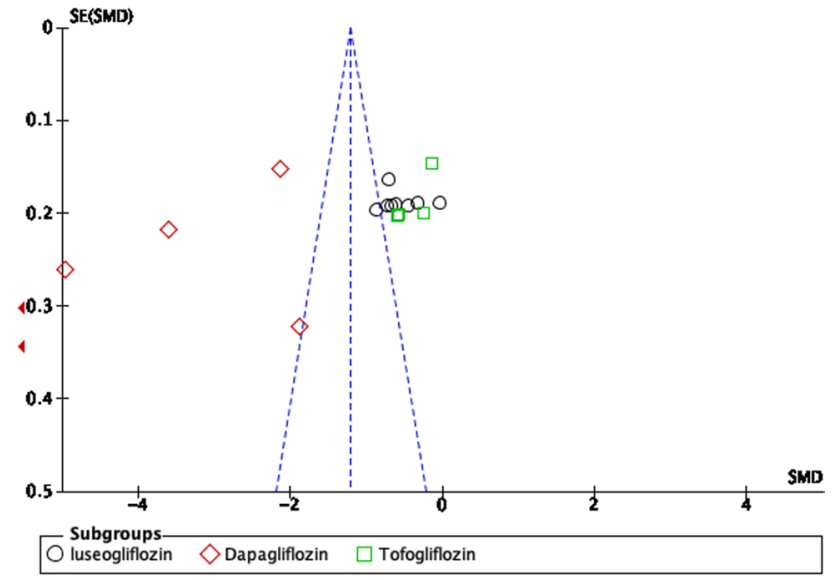

Fig. 9 Funnel plot of comparison in UA

consistent with a global meta-analysis of renal benefits that indicated the advantages of SGLT2 inhibitors in delaying the decline of eGFR and reducing Scr [40]. The mechanism through which dapagliflozin protects the renal system is unclear and may be related to the reduction of glomerular filtration. Some scientists believe that SGLT2 inhibitors may increase erythrocyte-specific volume, improve the hypoxic state of renal tubules, partially reverse renal tubule injury, and delay the progression of DKD [41]. Glucose reabsorption in the renal tubules is considered to be the most important mechanism for regulating blood glucose homeostasis, which is mainly regulated by the proximal convoluted tubules and the

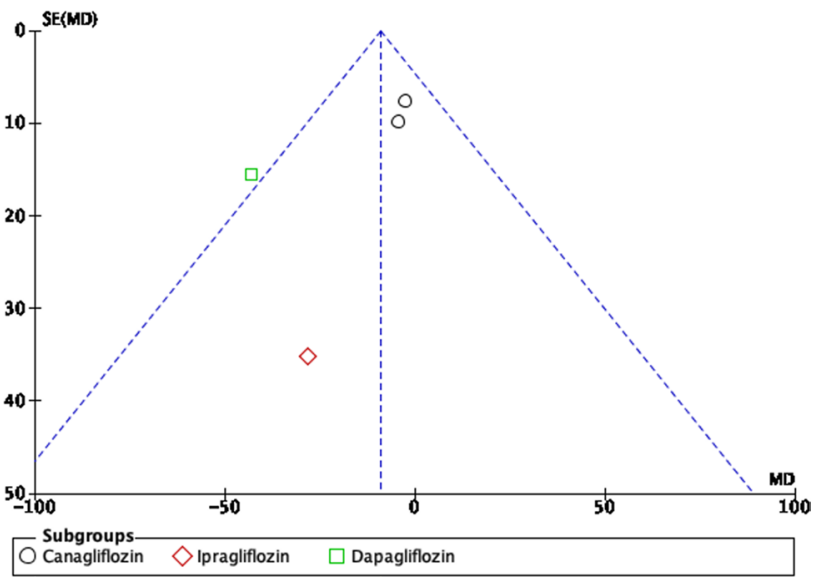

Fig. 11 Funnel plot of comparison in UACR

sodium-glucose cotransporter [4]. Among the 6 SGLT superfamilies, SGLT2 plays a leading role in glucose reabsorption. Both T1DM and T2DM models have confirmed that hyperglycemia in vivo stimulates the upregulation of SGLT2 receptors in the proximal tubules, leading to increased sodium ions reabsorption in the proximal tubules. These changes lead to a decrease of $\mathrm{NA}^{+}$in the distal tubule fluid, activation of dense plaques, increased GFR, and finally, a cascade reaction [42]. Many animal experiments and clinical trials have established that diabetes leads to inappropriate activation of renal renin-angiotensin-aldosterone system (RAAS), which

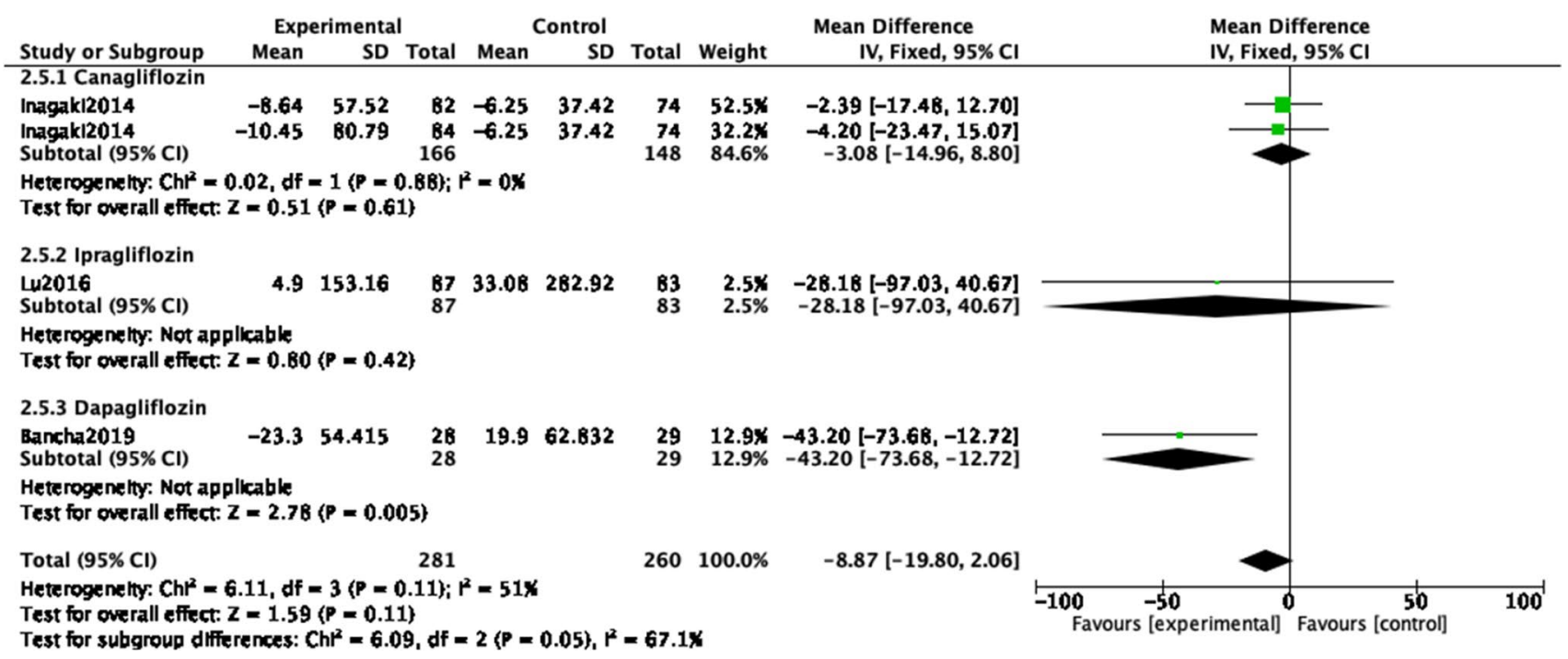

Fig. 10 Forest plot of the \% change in the urinary microalbumin/creatinine ratio (UACR) in the patients randomized to SGLT2 inhibitor therapy versus control therapy. SD standard deviation, CI confidence interval, IV inverse variance 


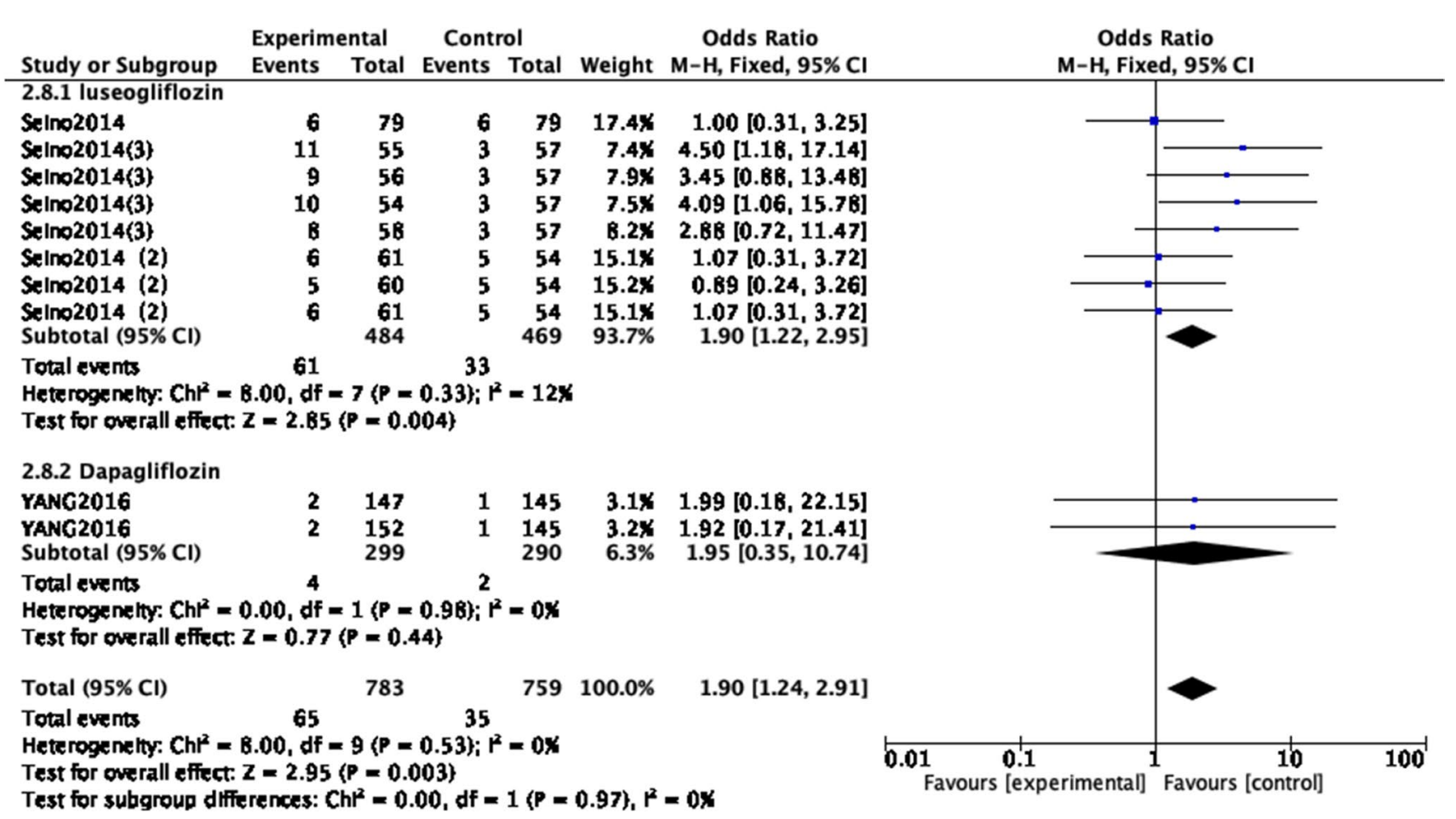

Fig. 12 Forest plot of the \% change in AEs related to renal function in the patients randomized to SGLT2 inhibitor therapy versus control therapy. SD standard deviation, CI confidence interval, IV inverse variance

is an important mechanism for the occurrence of DKD [43]. Increased SGLT2 activity leads to increased reabsorption of dense plaques and decreased $\mathrm{NaCl}$ concentration and stimulates the release of renin from accessory cells. High glucose promotes angiotensinogen (AGT) production, which activates RAAS in the kidneys [44]. Studies have shown that the mechanism of UA reduction may be related to the activation of uric acid transporter

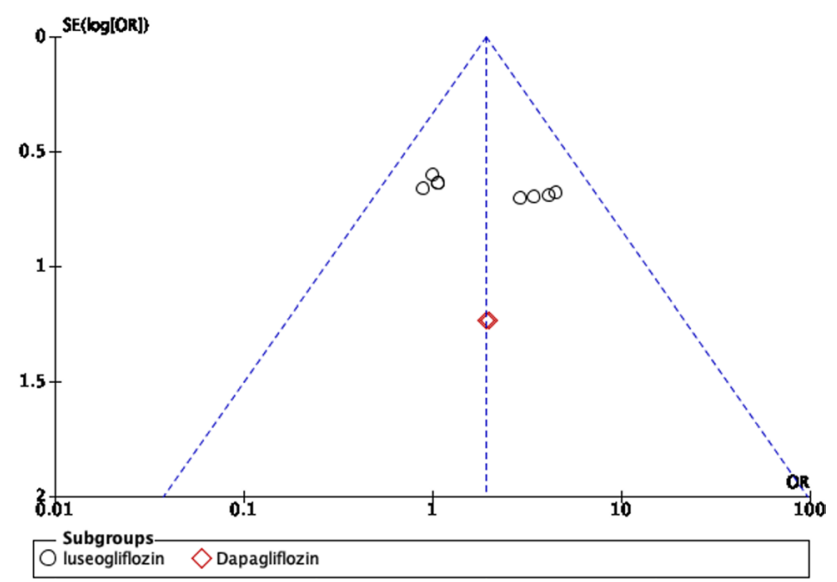

Fig. 13 Funnel plot of comparison in AEs related to renal function
9 (glucose induced by GLUT9 and SLC2A9) and other transporters [45]. Although SGLT2 inhibitors reduce UA by increasing UA excretion, past studies did not find an increase in the incidence of kidney stones [46].

A previous global meta-analysis showed that compared with other traditional therapies or placebos, SGLT2 inhibitors may reduce albuminuria and significantly benefit renal composite endpoint events [47]. They may also improve renal hemodynamics, reduce glomerular ultrafiltration and intrarenal pressure, and reduce albuminuria [41]. These findings of a protective effect of SGLT2 inhibitors on the renal system are consistent with the trends found in our study's results, but we also found differences among our population of Asian patients. Specifically, we determined that the use of SGLT2 inhibitors in Asian patients with T2DM is not associated with reductions in albuminuria. Still, dapagliflozin seems to have some effects on reducing albuminuria, although its mechanism is not yet clear, and more clinical studies are needed to verify this effect. We also found that while SGLT2 inhibitors may increase AEs related to renal function, they did not increase the incidence of renal impairment. Further, the reported AEs were classified as mild-to-moderate and were predominantly resolved during the course of the studies. The mechanism by which 


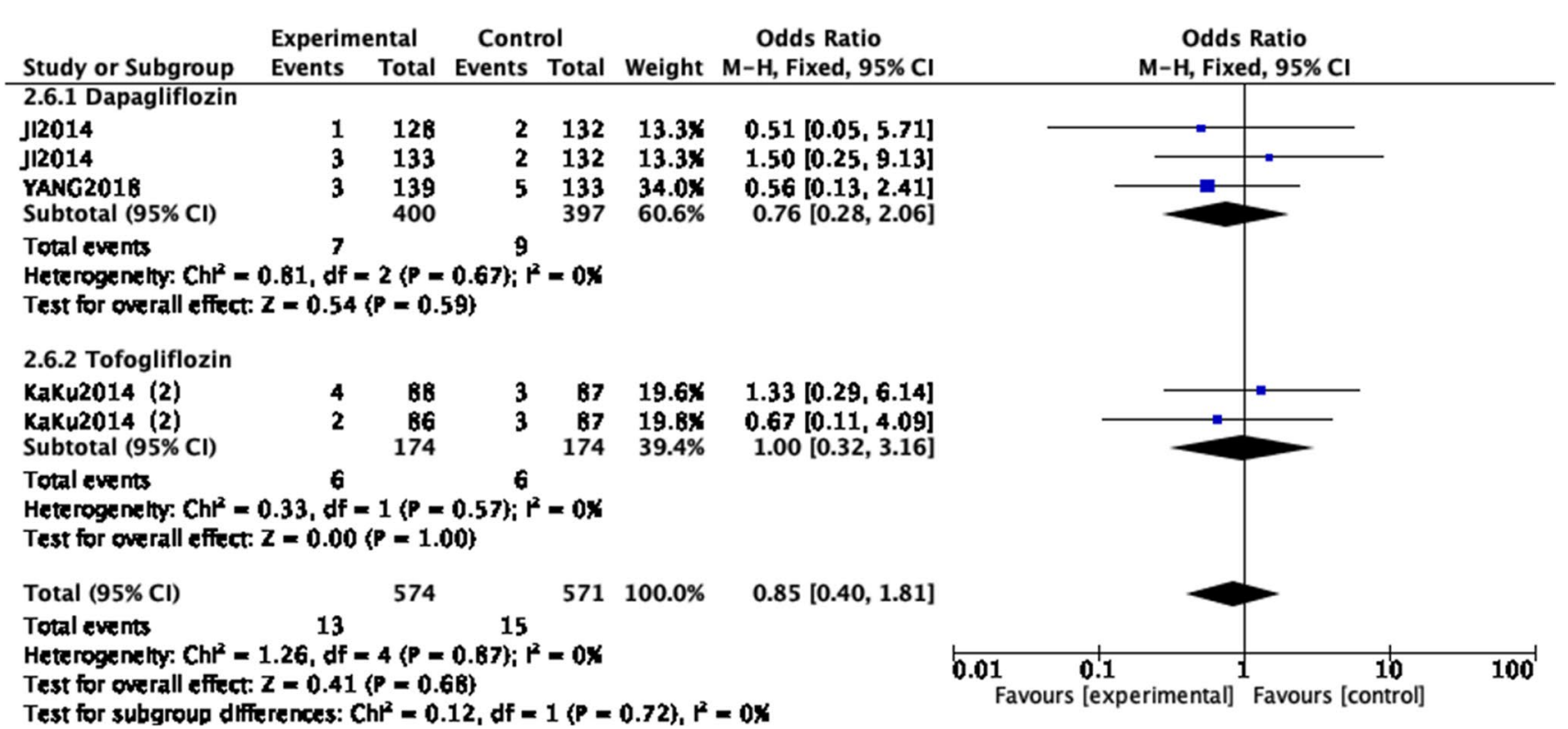

Fig. 14 Forest plot of the \% change in renal impairment in the patients randomized to SGLT2 inhibitor therapy versus control therapy. SD standard deviation, CI confidence interval, IV inverse variance

short-term SGLT2 inhibitor use causes transient AEs in Asians is unclear and warrants further study. More RCTs are also needed to study the impact of SGLT2 inhibitors in Asians, apply it to patients with lower levels of eGFR, especially apply it to patients with the CKD.

This study has some limitations. First, the included RCTs only investigated short-term ( $<24$ weeks) outcomes. Second, the renal outcomes of the included studies were heterogeneous. This may have been related to variation in population baselines, drugs, doses of the same drug, study duration, and definitions of and methods used to collect outcomes.

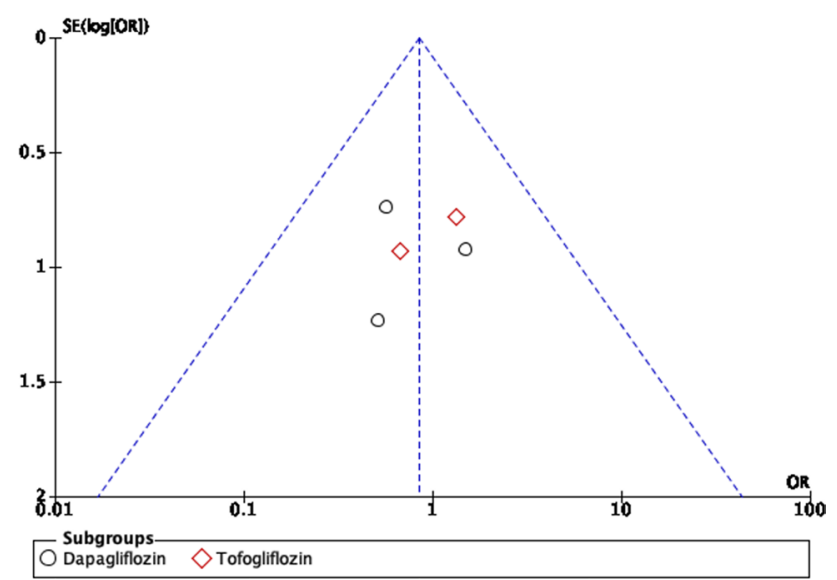

Fig. 15 Funnel plot of comparison in renal impairment
Finally, the included RCTs likely had a publication bias as positive results are more likely to be published. More clinical RCTs focused on renal events are needed, especially in populations with a relatively low GFR and in patients without albuminuria.

In conclusion, the use of SGLT2 inhibitors in Asian patients with T2DM may delay the decline of eGFR and reduce Scr and UA. Although SGLT2 inhibitors have no overall advantage in reducing albuminuria, dapagliflozin does appear to reduce albuminuria. Finally, SGLT2 inhibitors may increase the incidence of AEs related to renal function, but do not increase the incidence of renal impairment. More high-quality RCTs are needed to establish the benefits of SGLT2 inhibitors on the renal system and to better guide clinical medication.

Funding The current study was funded by the National Natural Science Foundation of China Project (81600565) and the Outstanding Youth Program of Jiangsu Natural Science Foundation Project (BK 20190052).

\section{Declarations}

Conflict of interest The authors declare no competing interests.

Open Access This article is licensed under a Creative Commons Attribution 4.0 International License, which permits use, sharing, adaptation, distribution and reproduction in any medium or format, as long as you give appropriate credit to the original author(s) and the source, 
provide a link to the Creative Commons licence, and indicate if changes were made. The images or other third party material in this article are included in the article's Creative Commons licence, unless indicated otherwise in a credit line to the material. If material is not included in the article's Creative Commons licence and your intended use is not permitted by statutory regulation or exceeds the permitted use, you will need to obtain permission directly from the copyright holder. To view a copy of this licence, visit http://creativecommons.org/licenses/by/4.0/.

\section{References}

1. H C N, E S J, S K, et al. IDF diabetes atlas: global estimates of diabetes prevalence for 2017 and projections for 2045. Diabetes research and clinical practice, 2018,138 .

2. Nelson R G, Newman J M, Knowler W C, et al. Incidence of end-stage renal disease in type 2 (non-insulin-dependent) diabetes mellitus in Pima Indians. Diabetologia, 1988,31(10).

3. Ma R C W, Chan J C N. Type 2 diabetes in East Asians: similarities and differences with populations in Europe and the United States . Annals of the New York Academy of Sciences, 2013,1281(1)

4. Andrianesis V, Doupis J. The role of kidney in glucose homeostasis-SGLT2 inhibitors, a new approach in diabetes treatment. Expert Rev Clin Pharmacol. 2013;6(5):519-39.

5. Zelniker TA, Wiviott SD, Raz I, et al. SGLT2 inhibitors for primary and secondary prevention of cardiovascular and renal outcomes in type 2 diabetes: a systematic review and meta-analysis of cardiovascular outcome trials. Lancet. 2019;393(10166):31-9.

6. Jadad AR, Moore RA, Carroll D, et al. Assessing the quality of reports of randomized clinical trials: is blinding necessary? Control Clin Trials. 1996;17(1):1-12.

7. Yang W, Ma J, Li Y, et al. Dapagliflozin as add-on therapy in Asian patients with type 2 diabetes inadequately controlled on insulin with or without oral antihyperglycemic drugs: a randomized controlled trial. J Diabetes. 2018;10(7):589-99.

8. Kaku K, Kiyosue A, Inoue S, et al. Efficacy and safety of dapagliflozin monotherapy in Japanese patients with type 2 diabetes inadequately controlled by diet and exercise. Diabetes Obes Metab. 2014;16(11):1102-10.

9. Inagaki N, Kondo K, Yoshinari T, et al. Efficacy and safety of canagliflozin monotherapy in Japanese patients with type 2 diabetes inadequately controlled with diet and exercise: a 24-week, randomized, double-blind, placebo-controlled, phase III study. Expert Opin Pharmacother. 2014;15(11):1501-15.

10. Kaku K, Watada H, Iwamoto Y, et al. Efficacy and safety of monotherapy with the novel sodium/glucose cotransporter-2 inhibitor tofogliflozin in Japanese patients with type 2 diabetes mellitus: a combined phase 2 and 3 randomized, placebo-controlled, doubleblind, parallel-group comparative study . Cardiovasc Diabetol, 2014,13:65.

11. Satirapoj B, Korkiatpitak P, Supasyndh O. Effect of sodium-glucose cotransporter 2 inhibitor on proximal tubular function and injury in patients with type 2 diabetes: a randomized controlled trial. Clin Kidney J. 2019;12(3):326-32.

12. Seino Y, Sasaki T, Fukatsu A, et al. Efficacy and safety of luseogliflozin as monotherapy in Japanese patients with type 2 diabetes mellitus: a randomized, double-blind, placebo-controlled, phase 3 study. Curr Med Res Opin. 2014;30(7):1245-55.

13. Han KA, Chon $\mathrm{S}$, Chung $\mathrm{CH}$, et al. Efficacy and safety of ipragliflozin as an add-on therapy to sitagliptin and metformin in Korean patients with inadequately controlled type 2 diabetes mellitus: a randomized controlled trial. Diabetes Obes Metab. 2018;20(10):2408-15.
14. Ji L, Han P, Liu Y, et al. Canagliflozin in Asian patients with type 2 diabetes on metformin alone or metformin in combination with sulphonylurea. Diabetes Obes Metab. 2015;17(1):23-31.

15. Ji L, Ma J, Li H, et al. Dapagliflozin as monotherapy in drug-naive Asian patients with type 2 diabetes mellitus: a randomized, blinded, prospective phase III study. Clin Ther. 2014;36(1):84-100.

16. Lu CH, Min KW, Chuang LM, et al. Efficacy, safety, and tolerability of ipragliflozin in Asian patients with type 2 diabetes mellitus and inadequate glycemic control with metformin: results of a phase 3 randomized, placebo-controlled, double-blind, multicenter trial. J Diabetes Investig. 2016;7(3):366-73.

17. Terauchi Y, Tamura M, Senda M, et al. Efficacy and safety of tofogliflozin in Japanese patients with type 2 diabetes mellitus with inadequate glycaemic control on insulin therapy (J-STEP/INS): results of a 16-week randomized, double-blind, placebo-controlled multicentre trial. Diabetes Obes Metab. 2017;19(10):1397-407.

18. Seino Y, Sasaki T, Fukatsu A, et al. Dose-finding study of luseogliflozin in Japanese patients with type 2 diabetes mellitus: a 12-week, randomized, double-blind, placebo-controlled, phase II study. Curr Med Res Opin. 2014;30(7):1231-44.

19. Yang W, Han P, Min KW, et al. Efficacy and safety of dapagliflozin in Asian patients with type 2 diabetes after metformin failure: a randomized controlled trial. J Diabetes. 2016;8(6):796-808.

20. Seino Y, Sasaki T, Fukatsu A, et al. Efficacy and safety of luseogliflozin monotherapy in Japanese patients with type 2 diabetes mellitus: a 12-week, randomized, placebo-controlled, phase II study. Curr Med Res Opin. 2014;30(7):1219-30.

21. Holman N, Young B, Gadsby R. Current prevalence of type 1 and type 2 diabetes in adults and children in the UK. Diabet Med. 2015;32(9):1119-20.

22. Fu H, Liu S, Bastacky SI, et al. Diabetic kidney diseases revisited: a new perspective for a new era. Mol Metab. 2019;30:250-63.

23. Chan J C N, Malik V, Jia W, et al. Diabetes in Asia: epidemiology, risk factors, and pathophysiology . JAMA: The Journal of the American Medical Association, 2009,301(20).

24. Hu FB. Globalization of diabetes: the role of diet, lifestyle, and genes. Diabetes Care. 2011;34(6):1249-57.

25. Sjoblad S. Could the high consumption of high glycaemic index carbohydrates and sugars, associated with the nutritional transition to the Western type of diet, be the common cause of the obesity epidemic and the worldwide increasing incidences of type 1 and type 2 diabetes? Med Hypotheses. 2019;125:41-50.

26. Woodward M, Patel A, Zoungas S, et al. Does glycemic control offer similar benefits among patients with diabetes in different regions of the world? Results from the ADVANCE trial. Diabetes Care. 2011;34(12):2491-5.

27. Doria A, Warram JH, Krolewski AS. Genetic susceptibility to nephropathy in insulin-dependent diabetes: from epidemiology to molecular genetics. Diabetes Metab Rev. 1995;11(4):287-314.

28. Zhang R, Zhuang L, Li M, et al. Arg913Gln of SLC12A3 gene promotes development and progression of end-stage renal disease in Chinese type 2 diabetes mellitus. Mol Cell Biochem. 2018;437(1-2):203-10.

29. Fioretto P, Zambon A, Rossato M, et al. SGLT2 inhibitors and the diabetic kidney. Diabetes Care. 2016;39(Suppl 2):S165-71.

30. Bays HE, Weinstein R, Law G, et al. Canagliflozin: effects in overweight and obese subjects without diabetes mellitus. Obesity (Silver Spring). 2014;22(4):1042-9.

31. Ramirez-Rodriguez AM, Gonzalez-Ortiz M, Martinez-Abundis E. Effect of dapagliflozin on insulin secretion and insulin sensitivity in patients with prediabetes. Exp Clin Endocrinol Diabetes. 2020;128(8):506-11.

32. Hollander P, Bays HE, Rosenstock J, et al. Coadministration of canagliflozin and phentermine for weight management in overweight and obese individuals without diabetes: a randomized clinical trial. Diabetes Care. 2017;40(5):632-9. 
33. Lundkvist P, Sjostrom CD, Amini S, et al. Dapagliflozin oncedaily and exenatide once-weekly dual therapy: a 24 -week randomized, placebo-controlled, phase II study examining effects on body weight and prediabetes in obese adults without diabetes. Diabetes Obes Metab. 2017;19(1):49-60.

34. Heerspink H, Stefansson BV, Correa-Rotter R, et al. Dapagliflozin in patients with chronic kidney disease. N Engl J Med. 2020;383(15):1436-46.

35. D. S D W M, D. I R M, H. M P B M, et al. Dapagliflozin and cardiovascular outcomes in type 2 diabetes. New England Journal of Medicine, 2019,380(4).

36. Wanner C, Inzucchi SE, Lachin JM, et al. Empagliflozin and progression of kidney disease in type 2 diabetes. N Engl J Med. 2016;375(4):323-34.

37. Neal B, Perkovic V, Mahaffey KW, et al. Canagliflozin and cardiovascular and renal events in type 2 diabetes. N Engl J Med. 2017;377(7):644-57.

38. Perkovic V, Jardine MJ, Neal B, et al. Canagliflozin and renal outcomes in type 2 diabetes and nephropathy. N Engl J Med. 2019;380(24):2295-306.

39. Singh AK, Singh R. Cardiovascular outcomes with SGLT-2 inhibitors and GLP-1 receptor agonist in Asians with type 2 diabetes: a systematic review and meta-analysis of cardiovascular outcome trials. Diabetes Metab Syndr. 2020;14(4):715-22.

40. Neuen BL, Young T, Heerspink H, et al. SGLT2 inhibitors for the prevention of kidney failure in patients with type 2 diabetes: a systematic review and meta-analysis. Lancet Diabetes Endocrinol. 2019;7(11):845-54.

41. Sano M, Takei M, Shiraishi Y, et al. Increased hematocrit during sodium-glucose cotransporter 2 inhibitor therapy indicates recovery of tubulointerstitial function in diabetic kidneys. J Clin Med Res. 2016;8(12):844-7.

42. Arakawa K, Ishihara T, Oku A, et al. Improved diabetic syndrome in $\mathrm{C} 57 \mathrm{BL} / \mathrm{KsJ}-\mathrm{db} / \mathrm{db}$ mice by oral administration of the $\mathrm{Na}(+)$-glucose cotransporter inhibitor T-1095. Br J Pharmacol. 2001;132(2):578-86.

43. Gurley SB, Coffman TM. The renin-angiotensin system and diabetic nephropathy. Semin Nephrol. 2007;27(2):144-52.

44. Traynor TR, Smart A, Briggs JP, et al. Inhibition of macula densa-stimulated renin secretion by pharmacological blockade of cyclooxygenase-2. Am J Physiol. 1999;277(5):F706-10.

45. Davies MJ, Trujillo A, Vijapurkar U, et al. Effect of canagliflozin on serum uric acid in patients with type 2 diabetes mellitus. Diabetes Obes Metab. 2015;17(4):426-9.

46. Bolinder J, Ljunggren $\mathrm{O}$, Kullberg $\mathrm{J}$, et al. Effects of dapagliflozin on body weight, total fat mass, and regional adipose tissue distribution in patients with type 2 diabetes mellitus with inadequate glycemic control on metformin. J Clin Endocrinol Metab. 2012;97(3):1020-31.

47. Luo Y, Lu K, Liu G, et al. The effects of novel antidiabetic drugs on albuminuria in type 2 diabetes mellitus: a systematic review and meta-analysis of randomized controlled trials. Clin Drug Investig. 2018;38(12):1089-108.

Publisher's note Springer Nature remains neutral with regard to jurisdictional claims in published maps and institutional affiliations. 